Practical Considerations for High Spatial and Temporal Resolution Dynamic Transmission Electron Microscopy

Michael Armstrong, Ken Boyden, Nigel D. Browning, Geoffrey H. Campbell, Jeffrey D. Colvin, Bill DeHope, Alan M. Frank, David J. Gibson, Fred Hartemann, Judy S. Kim, Wayne E. King, Thomas B. LaGrange, Ben J. Pyke, Bryan W. Reed, Richard M. Shuttlesworth, Brent C. Stuart, Ben R. Torralva

May 3, 2006

Ultramicroscopy 
This document was prepared as an account of work sponsored by an agency of the United States Government. Neither the United States Government nor the University of California nor any of their employees, makes any warranty, express or implied, or assumes any legal liability or responsibility for the accuracy, completeness, or usefulness of any information, apparatus, product, or process disclosed, or represents that its use would not infringe privately owned rights. Reference herein to any specific commercial product, process, or service by trade name, trademark, manufacturer, or otherwise, does not necessarily constitute or imply its endorsement, recommendation, or favoring by the United States Government or the University of California. The views and opinions of authors expressed herein do not necessarily state or reflect those of the United States Government or the University of California, and shall not be used for advertising or product endorsement purposes. 


\title{
Practical Considerations for High Spatial and Temporal Resolution Dynamic Transmission Electron Microscopy
}

\author{
Michael R. Armstrong ${ }^{\dagger}$, Ken Boyden ${ }^{\dagger}$, Nigel D. Browning ${ }^{\dagger \dagger}$, Geoffrey H. Campbell ${ }^{\dagger}$, \\ Jeffrey D. Colvin ${ }^{\dagger}$, William J. DeHope ${ }^{\dagger}$, Alan M. Frank ${ }^{\dagger}$, David J. Gibson ${ }^{*}$, Fred Hartemann ${ }^{*}$, \\ Judy S. Kim ${ }^{\dagger \dagger}$, Wayne E. King ${ }^{\dagger}$, Thomas B. LaGrange ${ }^{\dagger}$, Ben J. Pyke ${ }^{\dagger}$, Bryan W. Reed ${ }^{\dagger}$, \\ Richard M. Shuttlesworth $^{\dagger}$, Brent C. Stuart ${ }^{\dagger}$, and Ben R. Torralva ${ }^{\dagger}$ \\ ${ }^{\dagger}$ Materials Science and Technology Division, Chemistry and Materials Science Directorate, \\ Lawrence Livermore National Laboratory, P.O. Box 808; L-356, Livermore, CA 94550 \\ * Division, Physics and Advanced Technologies Directorate, Lawrence Livermore National \\ Laboratory, P.O. Box 808; L-280, Livermore, CA 94550 \\ ${ }^{\ddagger}$ Department of Chemical Engineering and Materials Science, University of California-Davis, \\ One Shields Ave, Davis, CA 95616
}

\begin{abstract}
$\underline{\text { Abstract }}$
Although recent years have seen significant advances in the spatial resolution possible in the transmission electron microscope (TEM), the temporal resolution of most microscopes is limited to video rate at best. This lack of temporal resolution means that our understanding of dynamic processes in materials is extremely limited. High temporal resolution in the TEM can be achieved, however, by replacing the normal thermionic or field emission source with a photoemission source. In this case the temporal resolution is limited only by the ability to create a short pulse of photoexcited electrons in the source, and this can be as short as a few femtoseconds. The operation of the photo-emission source and the control of the subsequent pulse of electrons (containing as many as $5 \times 10^{7}$ electrons) create significant challenges for a standard microscope column that is designed to operate with a single electron in the column at any one time. In this paper, the generation and control of electron pulses in the TEM to obtain a temporal resolution $<10^{-6} \mathrm{~s}$ will be described and the effect of the pulse duration and current density on the spatial resolution of the instrument will be examined. The potential of these levels of temporal and spatial resolution for the study of dynamic materials processes will also be discussed.
\end{abstract}

PACS codes: 68.37.Lp, 07.78.+s, 78.47.+p

Keywords : Electron Microscope design and characterization, Electron sources, Particle optics

Released under LLNL information management, UCRL-JRNL-221064 


\section{Introduction}

Recently there have been several developments in transmission electron microscopy (TEM) that have pushed spatial resolution and sensitivity to the single atom level for imaging, diffraction and spectroscopy [1-6]. These new hardware and software capabilities have significantly extended our understanding of materials properties, particularly for nanoscale materials. However, throughout these developments, one area that has remained relatively unexplored has been the measurement of atomic processes on very short to ultra-short timescales $\left(10^{-6}-10^{-15} \mathrm{~s}\right)$. With few exceptions, time resolved studies have remained largely within the purview of optical and x-ray synchrotron methods.

While femtosecond spectroscopy and pump-probe experiments using advanced laser and synchrotron technology have proved to be very successful [7-10], there are several advantages to using electron pulses on the same timescale to analyze materials. The immediate advantage is that the experiments can be performed in conjunction with current TEM methods for materials characterization - here TEM is the only experimental method that can directly generate nanometer-resolution real space images of the sample on these timescales. Secondly, electrons can be focused to a probe of atomic dimensions. Finally, the interaction of the electrons with the material being analyzed is much stronger compared to x-rays, producing more signal. Thus, if high time resolution can be brought to the TEM, existing analytical techniques of electron diffraction, imaging and spectroscopy could be employed with both the highest spatial and time resolution available to any method of analysis [12].

In this paper, we outline the modifications of a standard TEM that are needed to generate, control and utilize short pulses at the highest spatial resolution (Section 2). We discuss the relative merits of the two approaches and the effect of large numbers of electrons in a single 
pulse on the expected resolution of the microscope in Section 3. The first DTEM results at Lawrence Livermore National Laboratory (LLNL) to achieve $\sim$ nanosecond and $\sim$ nanometer resolution from a single pulse of electrons will be described at the end of Section 3 and the potential future applications of these methods will be discussed in Section 4.

\section{Modifying the Standard TEM}

The principle behind the observation of dynamic processes in TEM is illustrated in Figure 1. A sample is excited by an initiating laser pulse and then examined by a later analytical pulse of electrons. By studying the sample before and after the arrival of the initiation pulse, one may obtain information on many aspects of the dynamic or transient properties. The sensitivity of the measurement is, as with most TEM studies, governed by the signal-to-noise in the image and/or diffraction pattern. In order to observe transient processes, it is essential to either generate enough electrons within a single pulse or study a reversible process where the scattering from many pulses can be summed to obtain high enough signal for the best resolution of the microscope.

One of these approaches, the multiple shot or stroboscopic approach, involves rapidly repeating the experiment on the same region of sample, or rapidly refreshed sample, and summing the results. Stroboscopic acquisition enables rapid averaging of data from many experiments and is necessarily an ensemble measurement. In the mid-1970s, Bostanjoglo and Rosin [13] developed an early capability to produce stroboscopic images in TEM using beam blanking. Recently, Zewail et al. $[14,15]$ have pushed the stroboscopic method to the extreme with a modern implementation employing femtosecond lasers. Each electron pulse has on average only one electron, thereby eliminating space charge effects and enabling imaging 
resolution equivalent to that of conventional TEM. Images are built up from millions of singleelectron pulses that have been precisely correlated with the specimen drive laser. This method allows temporal and spatial resolution to be maintained at the optimum levels, but the fact that the specimen must be laser-pumped millions of times means that the process being studied must be repeatable or that the sample must completely recover between shots.

The single shot approach to achieving high time resolution in TEM was pioneered by Bostanjoglo and co-workers and aims to generate pulses with enough electrons to form interpretable images from a single pulse of electrons $[16,17]$. The single shot approach means that the process being studied need not be perfectly repeatable as all the information is obtained from a single specimen drive event. However, the limitation to this method is that space-charge effects in the beam can lead to degradation of resolution and even with an optimized microscope source, column, and detector the high current will limit the overall temporal and spatial resolution of the instrument. The key to using this single shot approach is therefore to optimize the components in the microscope to define the space-charge limited resolution of the instrument.

The principles governing the design and operation of a dynamic TEM (DTEM) for either the single shot or stroboscopic approach to obtaining high time resolution are essentially the same. The only difference between the two approaches is in the optimization of the column design to reduce space charge effects.

The DTEM at LLNL consists of a JEOL 2000FX that has been modified as shown in the Figure 1. A section has been added to the microscope column below the electron gun. This section includes a window to bring a frequency quintupled Nd:YLF, $211 \mathrm{~nm}$ wavelength laser 
pulse into the TEM column and a mirror in the center of this section to direct the laser photoexcitation pulse to an $840 \mu \mathrm{m}$ tantalum disk cathode. The laser pulse photoexcites an electron pulse from the cathode of 30-35 ns FWHM duration. This electron pulse accelerates through the electron gun and passes through a hole in the mirror to continue to the condenser, sample, and projection sections as in standard TEM. The DTEM has also been modified so that a separate laser pulse excites the sample. A pump-probe experiment in the DTEM starts with the excitation of the sample by a drive laser pulse, followed by probing the sample with an electron pulse with post-sample lenses set in either diffraction or imaging mode. Several experiments are done with different time delays between the pump and probe, which are used to construct a "movie" of the evolving dynamics of the sample.

A schematic of the laser system is shown in Figure 2. For nanosecond time scale experiments, the laser consists of two Q-switched neodymium based lasers with emission wavelengths of $1064 \mathrm{~nm}$ and $1053 \mathrm{~nm}$ and pulse durations that depend on the design of the laser. Often, for optical pump-probe experiments the time delay is generated using an optical delay line, but this technique requires 300 meters of distance per $\mu$ s of delay time and is impractical for microsecond delays. We obtain microsecond time delays by activating the two lasers with electronically delayed trigger pulses. In our case, the pump laser has a pulse duration of around $10 \mathrm{~ns}$ and the cathode (probe) laser has a pulse duration of around $70 \mathrm{~ns}$. For efficient photoemission, the cathode pulse is frequency converted to obtain $211 \mathrm{~nm}\left(5^{\text {th }}\right.$ harmonic $)$ light. This wavelength is above the work function for many materials, and allows us to change the cathode material without changing the laser wavelength. Since frequency conversion is a nonlinear process, the duration of the $211 \mathrm{~nm}$ pulse is shorter than the fundamental pulse, around 30- 
$35 \mathrm{~ns}$ in our case. Depending on the sample, the pump pulse may also be frequency converted, but for metal samples with broadband absorption, often we use $1064 \mathrm{~nm}$ light.

The cathode laser pulse typically starts with about $200 \mathrm{~mJ}$ of energy, giving $<1 \mathrm{~mJ}$ of energy in the $5^{\text {th }}$ harmonic. We use about $200 \mu \mathrm{J}$ of energy incident on the cathode to obtain $\sim 3-4 * 10^{7}$ electrons per pulse. Given published values of the photoemission quantum efficiency (around 10 ${ }^{5}-10^{-4}$ ) [18], the electron yield with low laser excitation (i.e. no saturation) at the sample is 10100 times less than would be expected if every photoemitted electron reached the sample. We attribute this to inefficiency in collection of electrons in the gun/condenser system of the microscope. Typically, the pump laser generates 5-10 mJ of energy per pulse.

The LLNL DTEM was designed for single shot operation. The lasers generate relatively large energy per pulse and have low maximum pulse repetition rate $(\sim 10 \mathrm{~Hz})$. An experiment requires alignment in standard mode, optimization of the alignment in pulsed mode with the probe electron pulse operating at $10 \mathrm{~Hz}$, followed by an experiment with single shot pump and probe pulses. Since these experiments usually destroy or permanently alter the sample, the sample must be moved to another location for the next shot. Using this method, a series of shots are accumulated with different time delays between the pump and probe.

Finally, alignment of both the cathode and pump laser beams is critical in the operation of DTEM. The confined, mostly optically inaccessible DTEM column does not lend itself to standard optical alignment techniques, so laser alignment must be carefully considered in any DTEM design.

\section{Practical Resolution Limits}


The alignment and operation of the DTEM is essentially determined by the need to have a high enough signal to noise ratio in the final image to observe structural features at high spatial resolution (here the stroboscopic approach simply sums a number of observations to form the final image, potentially relaxing the signal to noise constraints considerably if the number of reversible events that can be sampled is large). The minimum number of electrons needed for a single shot DTEM image can be determined by assuming a rough constraint for distinguishing pixels in an image:

$$
\text { Shot noise } \leq \frac{\text { contrast }}{\# \text { of grey scale levels }}
$$

For a useful image, the minimum number of grey levels is chosen to be around 5.

$$
\text { The noise is given by: } \quad \text { Shot } \text { noise }=\frac{1}{\sqrt{N}}
$$

where $\mathrm{N}$ is the number of electrons detected per pixel.

$$
N=\text { time integrated electron fluence (e-/area) * pixel area }
$$

For $10^{7}$ electrons in a $1 \mu^{2}$ area $\left(10\right.$ electrons $\left./ \mathrm{nm}^{2}\right)$ and $50 \%$ contrast in a shot noise limited imaging system, the noise would have to be below 10\%, implying 100 electrons/pixel, and a resolution of $\sim 3 \mathrm{~nm}$. This analysis assumes that the imaging system can achieve this resolution (this will be discussed later in this section) and provides the most straightforward estimate for the minimum number of electrons per pulse for a desired image resolution and contrast.

A large number of electrons in a nanosecond duration pulse results in a very large peak current compared to a traditional TEM. For comparison, $10^{7}$ electrons in a 1 ns pulse corresponds to about $2 \mathrm{~mA}$ of peak current. Yet, a "high current" application like high 
throughput electron lithography typically operates at some $10 \mathrm{~s}$ of $\mu \mathrm{A}$ of current and traditional TEM typically operates at currents in the nA regime. As a result, the operation of a single shot pulsed electron imaging instrument must attempt to reduce the effects of Coulomb interactions at these very high peak currents. The effects of Coulomb interactions are manifest in every section of the DTEM, and some of these effects cannot be easily reduced to simple models. As a result, detailed modeling of the column will be necessary to optimize the design of future generations of DTEM. Some simple models can be applied that provide a rough guide to some of the problems and possible solutions.

The effects of Coulomb repulsion can be divided into two broad categories: stochastic blurring and global space charge. Stochastic blurring is the irreversible loss of image information encoded in an electron pulse via random scattering of electrons [19, 20], as illustrated in Figure 3 and will be discussed in Section 3.3.

Global space charge (GSC) is overall expansion of the electron bunch. Since global space charge expansion is a large-scale effect, it is more straightforward to model than stochastic blurring with models widely used in the particle accelerator community [21, 22]. An example of global space charge expansion based on a uniform ellipsoid model of the pulse is shown in Figure 4. For a uniform electron distribution, global space charge expansion will act like a perfect negative electron lens and may be compensated by standard electron optics in principle. Nonetheless, generally the pulse distribution will be non-uniform, so the effective GSC lens will be aberrated. [19, 23] Although the effects of global space charge are not as severe for pulses of nanoseconds and longer, they must still be compensated in the gun and condenser systems at all times, where electron generation and collection are critical. 


\subsection{Gun and Condenser System}

A major concern in the construction and operation of the DTEM has been the generation and delivery of electrons to illuminate the sample. Figure 5 shows the integrated charge per pulse as a function of cathode laser pulse energy for an early incarnation of the DTEM. Although pulse charge grows linearly with laser pulse energy for low energy, the charge quickly saturates with increasing laser energy. Also, the slope efficiency of the graph in the linear regime is less by a factor of 100 than the expected value given the photoemission quantum efficiency for tantalum [18]. Essentially, these issues comprise at least three effects: 1) limits to the generation of high brightness electron pulses due to saturation of current at the cathode (the Child-Langmuir effect [24]), 2) GSC pulse expansion transverse to the electron beam axis, which limits the number of electrons collected by the condenser system, and 3) the intrinsic design of the JEOL 2000FX, which is optimized for direct current operation and obtains high beam quality at the sample by removing low quality electrons (i.e. far-from-paraxial electrons, which are subject to excess aberrations) from the beam with apertures. Although the design of the $2000 \mathrm{FX}$ may be responsible for the low collection efficiency at low laser excitation, non-linear saturation of the charge per pulse is due to the space charge effects described earlier. These effects limit the peak current that may be delivered to illuminate the sample. It should be noted that lengthening the duration of the electron pulse may always be used to obtain a higher number of electrons per pulse, at the expense of time resolution.

The Child-Langmuir effect limits the current that may be emitted from a cathode as a function of the cathode-anode separation and the potential difference, with higher current obtained with a higher potential drop and a shorter cathode-anode separation. The main approaches to mitigating this current limit in future DTEM designs are the same as have been 
developed for other high brightness electron sources [25, 26]: increase the electric field at the surface and decrease the acceleration gap. This may involve a shorter electron gun, a higher extraction field near the cathode, an RF accelerator [26], or eventually very advanced solutions like the use of a cooled gas to emit an ultracold gas of electrons [21]. Ultimately, the electron gun must generate both high peak current and high beam quality [27].

Simulations of uniform pulse propagation through the electron gun in the DTEM indicate that the space charge induced transverse (perpendicular to the beam axis) expansion of the electron pulse becomes significant for current above $2 \mathrm{~mA}$, which corresponds to about $10^{7}$ electrons in a $1 \mathrm{~ns}$ pulse, and dominates the transverse beam expansion as current approaches $200 \mathrm{~mA}$, for initial spot sizes at the cathode below $500 \mu \mathrm{m}$ in diameter. For a hundreds of $\mu \mathrm{m}$ collection aperture, this space charge expansion will limit the number of collected electrons by orders of magnitude. To optimize the collection of electrons by the condenser system, the effective aperture of the condenser systems must be increased and, to insure the preservation of a high quality electron beam through the condenser system, aberration corrected lenses would be preferred. Generally, reducing the propagation time of electrons from the cathode to the condenser system should improve electron collection. Our simulations also indicate that an extra lens just after the gun may significantly increase collection efficiency without increasing beam emittance.

The use of photoexcitation to generate electrons in the electron gun (in contrast to thermionic or field emission) may allow significant alteration of the cathode to optimize the beam quality or total charge per pulse. Since a photoexcited cathode can emit from a surface of arbitrary geometry, the cathode need not be a point emitter as in typical electron microscopes. Furthermore, the cathode material may be optimized for photoemission quantum efficiency 
rather than robustness at high temperature (which is required for thermionic cathodes). For example, Figure 6 shows a flat cathode design embedded in a typical electron accelerator. The electric field is perpendicular to the surface of the cathode and electrons are accelerated exactly along the beam axis. Although an optimum design will depend on the desired instrument capabilities, the design freedom afforded by the use of photoemission may significantly influence future designs of the DTEM gun section.

\subsection{The Specimen}

Optimization of the pump laser spot is critical to obtain quality data about the sample. Detailed diagnostics of the laser pump beam must be performed to correctly assess the effect of the pump laser pulse on the sample. For instance, for metal samples where typically all of the pump pulse is absorbed by the sample, the amount of pump energy and the thickness of the sample must be accurately known to obtain an estimate of the temperature increase. The temperature calculation is not straightforward in wedged shaped samples (typical TEM sample geometry) with strong thickness gradients. This constraint is relaxed somewhat in samples with low absorption, where the temperature increase will not depend on the thickness of the sample. In addition, the pump spot must be sufficiently intense to alter the sample in the desired manner, but large enough to uniformly heat over the profile of the electron probe beam. Since the electron beam may be focused to a much tighter spot $\left(\sim\right.$ some $\left.\mu \mathrm{m}^{2}\right)$, this is straightforward. Generally, we have used single squares of 100-200 mesh TEM grids as the typical sample region. Alignment of the pump with the probe on the sample is accomplished by laser drilling holes in sacrificial samples with illumination in standard low resolution TEM.

To obtain sufficient electron fluence at the sample given limitations in brightness, it has been necessary to use highly non-parallel electron beam illumination onto the sample in the DTEM. 
This has two potential detrimental effects: 1) loss of contrast due to an electron beam angular spread that is comparable to electron scattering angles from the sample, and 2) loss of resolution due to lens aberrations at high numerical aperture. Generally, we observe slight variation in the optimum focal length of the objective lens in pulsed mode compared to direct current operation.

\subsection{The Imaging System}

Stochastic blurring is irreversible loss of image information due to electron scattering, and can be a significant source of resolution degradation in imaging with short pulses of electrons. After an electron pulse has scattered from a sample, image information is encoded on electron trajectories. As the electron pulse propagates through the imaging system, Coulomb interactions randomly perturb electron trajectories, gradually washing out image information (as illustrated in Fig. 3). Since this process is essentially random, the lost image information cannot be recovered. This blurring process can be characterized by a blur profile indicating the spatial distribution of the blur. In simulations, a blur profile is constructed by computationally propagating an electron pulse through the optical systems and comparing (at the image plane) the positions of pulse electrons to where they would be if they followed unperturbed trajectories through the optical system. The blur profile is a histogram of the deviation of pulse electrons from their unperturbed positions in the image plane. [28] Although a blur profile is similar to a point spread function, it is not quite the same since a point spread function is a linear transfer function, but a blur profile depends nonlinearly on the beam properties. Since blur depends on precise details of many electrons trajectories, simulations to obtain blur profiles require significant computational resources.

Although the topic of stochastic blurring has been addressed in the context of electron lithography [28, 29], this effect has not been addressed for imaging with electron pulses. 
Essentially, stochastic blurring grows with the electron density in the pulse and the amount of time the pulse propagates. The effects of stochastic blurring cannot be completely eliminated, since there is at least one place - the sample - where the electron pulse must have high density. For conventional imaging, the back focal plane of the objective lens will also have high electron density as the weakly convergent electrons on the specimen are imaged into disks in reciprocal space. Thereafter, the diverging electron pulse becomes less dense and, after passing through stages of magnification, blurring becomes less significant on the scale of the magnified image. An example of a calculated blur profile is shown in Figure 7. A beam with higher peak current will exhibit higher blur for the same electron optical configuration.

Although blur probably cannot be eliminated for sub-nanosecond duration electrons pulses (assuming a goal of $<10 \mathrm{~nm}$ resolution), a number of strategies help to mitigate blur. The electron fluence must be balanced between the effects of blur at high fluence and loss of resolution due to signal to noise limits at low fluence. Optimal fluence is obtained when the resolution at the desired signal to noise matches the blur. Since the desired signal to noise depends on the contrast, optimal fluence depends on contrast obtainable in the sample.

With respect to instrument design, several design strategies may help mitigate stochastic blur. Generally, increasing the divergence of the electron beam will reduce density and blur. The trade off for higher divergence is in higher lens aberrations (which reduce the resolution) at higher numerical aperture, and the finite scattering divergence angle from the sample region, which puts an upper limit on the beam divergence depending on the desired contrast.

We have obtained blur estimates from a series of simulations that model a representative slice of the beam as a cloud of some $10^{4}$ electrons with pairwise Coulomb interactions. Although blur 
decreases with decreasing electron density, our simulations indicate that the blur rate is still significant before the electron pulse exits the objective lens for typical beam divergence $(<10-20$ $\mathrm{mrad})$ and objective focal length $(2.3 \mathrm{~mm})$, with an electron density consistent with $10^{7}$ electrons in $1 \mu \mathrm{m}^{2}$ at the sample $\left(10 \mathrm{e}^{-} / \mathrm{nm}^{2}\right.$ fluence), spread over a $1 \mathrm{~ns}$ pulse duration. Since the effect of blur will be smaller after the objective due to image magnification, the objective focal length and magnification must be optimized. If the objective focal length is too small, the blur rate downstream will be too high. If the objective focal length is too large, blur in the post-objective section will integrate to a large value due to the long propagation length. Once again, the optimal values depend on the sample (through sample dependent contrast) and the desired resolution.

In addition, for high divergence illumination, the correction of global space charge-induced beam aberrations, and usability of the instrument for different samples under different illumination conditions, it will be desirable to create an instrument with more degrees of freedom than a traditional TEM. For instance, adjustable aberration corrected lenses, both static and possibly with some dynamic character ${ }^{1}[30]$ would be useful, as well as the ability to adjust critical lens spacings. This is primarily to compensate for peak current dependent changes in the microscope.

\subsection{Initial Results with Nanosecond and Nanometer Resolution}

The current iteration of DTEM features 35 ns duration electron pulses, with greater than $10^{7}$ electrons per pulse. Currently, we obtain better than $20 \mathrm{~nm}$ spatial resolution in single shot mode, as illustrated in Figure 8, with roughly $5 \times 10^{6}$ electrons detected at the CCD. The sample in the figure consists of alternating layers of gold and carbon with spacings of 20 and $30 \mathrm{~nm}$. The

\footnotetext{
${ }^{1}$ For pulses with time correlated energy spread, the resolution may be improved by rapidly changing the strength of an electrostatic lens as the pulse passes through, in order to compensate for chromatic aberration.
} 
single shot image in the figure demonstrates at least $20 \mathrm{~nm}$ resolution and this is more clearly seen in an average of 50 shots. Currently the image resolution is noise limited and it is likely that higher resolution may be obtained with more detected electrons at the CCD.

\section{Current and Future DTEM Applications}

The design of the dynamic TEM employed at LLNL means that the instrument can be used in either single shot or stroboscopic mode; the different modes of operation simply require the lasers to either have a higher beam energy with a low repetition rate (single shot) or a lower beam energy with a higher repetition rate (stroboscopic mode). As discussed previously, each of these modes is suited to different dynamic observations. The single shot approach is useful for non-reversible dynamic behaviors such and structural phase transformations and, the nucleation, growth and mobility of dislocations, voids and even complex nanostructures. The stroboscopic mode is useful for the analysis of reversible processes such as electronic phase transitions and gas reaction dynamics. A few examples of the types of experiments that are being performed at LLNL will be discussed here. However, it should be noted that basically any dynamic experiment should be possible in the instrument within the limitations described above.

The first experiments that have been developed for the DTEM at LLNL include the studies of martensitic phase transformations [31-33], phase transformations in the molecular crystal HMX (nitramine octahydro-1,3,5,7-tetranitro-1,3,5,7-tetrazocine), and the speed of the solid-state reaction front in reactive multilayer foils (RMLFs).

For the solid-to-solid phase transformation, the aim has been to start with systems amenable to a temperature driven transformation and move on to pressure driven transformations. $\mathrm{Ti}$ is being studied since it undergoes a structure change from $\mathrm{HCP} \alpha$ phase to $\mathrm{BCC}, \beta$ phase upon 
heating above $1155 \mathrm{~K}$. This transition occurs by a rapid martensitic type transformation where the structural change to the BCC phase is accomplished by a simple shear strain of the HCP unit cell $[32,33]$. The transformation has been quantitatively tracked through a series of selected area diffraction patterns collected using different delays between the pump laser heating pulse and the electron probe pulse (Figure 9). One the important findings of these studies was that incubation times for nucleation are strongly dependent on grain size, i.e. Ti foils with an average grain size of $40 \mathrm{~nm}$ have incubation times of $\sim 100 \mathrm{~ns}$, while materials with $100 \mu \mathrm{m}$ grain sizes have incubation times on the order of 500 ns. This suggests that numerous grain boundaries in nanocrystalline materials act as strong defects and heterogeneous sites for nucleation, reducing the nucleation barrier energy and increasing the nucleation and transformation rate. Future experiments will use time-resolved bright-field imaging to study the grain size dependence on nucleation processes (i.e. observe nucleation sites and autocatalytic processes) and martensite/parent interface dynamics.

For HMX, the kinetics of the phase transformation have significant implications for accurate modeling of explosive loading of materials and safety. The nucleation and growth kinetics in the $\beta-\delta$ phase transition play a critical role in the mechanism of ignition. The $\beta-\delta$ phase transition in HMX is temperature driven, and involves a change of the molecule from a "chair" to a "boat" conformation, accompanied by a crystal structure change from monoclinic to hexagonal, also accompanied by a $7 \%$ volume change and significant changes in the crystal morphology (Figure 10). [34] The details of the nucleation and growth process currently are poorly understood and accurate modeling is difficult. [35-37] Furthermore, there is essentially no data that addresses molecular coordination in nucleation and growth processes, and these processes are fundamental to our understanding of solid-state chemical reactions, crystallization and biomechanics. 
Imaging experiments will provide a complete spatial picture of the nucleation and growth kinetics - a direct nanoscopic view of nucleation events and subsequent growth of phase transformed regions. Imaging data will provide a far more complete picture of nucleation and growth kinetics than is currently available.

The third topic of study involves rapid solid-state chemical reactions. Alternating nanoscale layers of metals that, upon initiation by an external energy source (heat or spark), react to form intermetallic compounds in an exothermic formation reaction are called reactive multilayer foils (RMLF). Since the intermetallic formation is an exothermic process, heat is generated into the surrounding foil volume causing a chain reaction of new phase formation that propagates along the interfaces up to the ends of the foil (Figure 11) [38, 39]. Since RMLFs produce extremely high heat over a small surface area, they are useful to join dissimilar materials such as a metal and ceramic, exposing only the contact surfaces to destabilizing heat. This reaction, however, can travel across the interface at high speeds of several meters per second [40], specifically depending on the material system and bilayer thickness. The nature of the intermetallic phase formation process and rate limiting mechanisms such as interface diffusion or kinetics remain unclear [41]. Studying the morphology, microstructure, and time scales of the transient intermetallic phases will give a detailed sequence of events in the foils and also clarify the general science of diffusion-controlled reactions.

For the applications described above, the aim has been to achieve the best spatial resolution possible with a time resolution of $\sim$ nanoseconds in single shot mode. However, the effects of Coulomb interactions in the beam are reduced significantly for pulse durations that are lengthened towards the microsecond timescale (while increasing the number of electrons per pulse). In such cases, it is expected that the spatial resolution of the microscope can approach 
the point resolution $(\sim 0.2 \mathrm{~nm})$ of the microscope working in its normal mode. This means that the DTEM can be used for in-situ (environmental cell) studies on the microsecond timescale making it several orders of magnitude quicker than current in-situ TEMs. Only the holder to perform the heating, cooling, straining, gas reaction, etc. is required. This higher spatial resolution is particularly useful for the study of nanomaterials, which are currently being developed for a wide variety of structural, electronic, optical and electronic applications [42-45]. The DTEM has an advantage over other dynamic methods in the study of these systems since DTEM allows measurements in both real and reciprocal spaces. Nanometers spatial with ultrafast temporal resolution make DTEM more suitable than x-rays for studying the dynamics of nanostructures.

As a final example of the types of studies that can be performed with the DTEM, we discuss one application of the stroboscopic mode. It has already been shown that ultrafast optical excitation can be used to manipulate the collective phase of correlated electron systems [46-52]. Photo-injection of electron-hole pairs is analogous to chemical doping and can induce a number of dynamic phenomena, such as reverse spin-Peierls transitions, insulator-to-metal switching of Mott insulators [53] and melting of charge/orbital order [54]. Charge/orbitally ordered checkerboard patterns, mesoscopic stripe formation, and similar electronic lattices are some of the examples of charge-ordering phenomena of interest. However, x-ray probing appears insufficient to fully study the dynamics of phase transitions, especially in complex oxides. An ambitious goal is the measurement of their dynamics with ultrafast time resolution by using time resolved electron diffraction. This is a difficult task with x-rays, due to their relatively low scattering cross section and to the unfavorable ratio of valence to core electrons in transition metals, as well as the substantial off-Bragg diffuse scattering. The high cross section of electrons 
makes this class of experiments possible by using the DTEM in stroboscopic mode, where stroboscopic mode is used to achieve the required high time resolution and may be used since the electronic phase transition is reversible.

\section{Conclusions}

The use of a photoemission source to obtain temporal resolution in the TEM allows images and diffraction patterns to be obtained with a time resolution between $\sim 10^{-6}$ and $\sim 10^{-15} \mathrm{~s}$. With the current generation of instruments, the spatial resolution of the final images is limited by space charge effects. The implementation of advanced designs for the various components of the microscope column offer the potential for resolution to be extended beyond the $\sim$ nanometer and $\sim$ nanosecond resolution of the current instrument. This high spatial and temporal resolution microscope has numerous potential applications for the study of structural and electronic phase transformations, catalytic activity in nanoparticles and nucleation and growth.

\section{Acknowledgements}

We would like to acknowledge the fantastic technical assistance of Rick Gross and Anne Bliss. We would also like to thank Ting Guo for helpful discussions concerning the applications of the DTEM and A. Cavalleri for his advice on electronic phase transition experiments to be performed on the DTEM in stroboscopic mode. Some electron gun simulations were performed using Trak 6.0 by Field Precision. Work was performed under the auspices of the U.S. Department of Energy by the University of California, Lawrence Livermore National Laboratory and supported by the Office of Science, Office of Basic Energy Sciences, Division of Materials Sciences and Engineering, of the U.S. Department of Energy under contract No. W-7405-Eng48. 


\section{References}

[1] P. E. Batson, N. Dellby, O. L. Krivanek, Nature 418 (2002) 617 .

[2] M. Haider, S. Uhlemann, E. Schwan, H. Rose, B. Kabius, K. Urban, Nature 392 (1998) 768.

[3] C. L. Jia, K. Urban, Science 303 (2004) 2001.

[4] P. D. Nellist, M. F. Chisholm, N. Dellby, O. L. Krivanek, M. F. Murfitt, Z. S. Zsilagyi, A. R. Lupini, A. Borisevich, W. H. Sides, S. J. Pennycook, Science 305 (2004) 1741.

[5] S. Lazar, G. A. Botton, M.-Y. Wu, F. D. Tichelaar, H. W. Zandbergen, Ultramicroscopy 96 (2003) 535.

[6] J. Bradley, Z. Dai, R. Erni, N. D. Browning, G. Graham, P. Weber, J. Smith, I. Hutcheon, H. Ishii, S. Bajt, C. Floss, F. Stadermann, Science 307 (2005) 244.

[7] C. Bressler, M. Chergui, Chem. Rev. 104 (2004) 1781-1812.

[8 J. R. Helliwell, P. M. Rentzepis, Oxford: Clarendon Press; New York: Oxford University Press, 1997.

[9] C. Rischel, A. Rousse, I. Uschmann, P. A. Albouy, J. P. Geindre, P. Audebert, J. C. Gauthier, E. Forster, J. L. Martin, A. Antonetti, Nature 390 (1997) 490-492.

[10] F. Raksi, K. R. Wilson, Z. M. Jiang, A. Ikhlef, C. Y. Cote, J.C. Kieffer, J. Chem. Phys. 104 (1996) 6066-6069.

[11] B.L. Weeks, C.M. Ruddle, J.M. Zaug, D.J. Cook, Ultramicroscopy 93 (2002) 19-23.

[12] W.E. King, G.H. Campbell, A. Frank, B. Reed, J.F. Schmerge, B.J. Siwick, B.C. Stuart, P.M. Weber, Journal of Applied Physics 97 (2005) 111101.

[13] O. Bostanjoglo, T. Rosin, Optica Acta 24 (1977) 657-664.

[14] J. Cao, H. Ihee, A.H. Zewail, Chemical Physics Letters 290 (1998) 1-8.

[15] A.H. Zewail, Philosophical Transactions of the Royal Society of London Series aMathematical Physical and Engineering Sciences 363 (2005) 315-329.

[16] O. Bostanjoglo, Advances in Imaging and Electron Physics, Vol 121121 (2002) 1-51.

[17] O. Bostanjoglo, W.R. Horinek, Optik 65 (1983) 361-367.

[18] W.E. King, M.R. Armstrong, O. Bostanjoglo, B.W. Reed, High Speed Electron Microscopy, in Science of Microscopy, P. Hawkes, J. Spence, Editors. 2006, Springer Verlag.

[19] G.H. Jansen, Coulomb Interactions in Particle Beams, San Diego, CA: Academic, 1990

[20] W.K. Waskiewicz, L.R. Harriott, J.A. Liddle, S.T. Stanton, S.D. Berger, E. Munro, X. Zhu, Microelectronic Engineering 42 (1998) 215-218.

[21] O.J. Luiten, S.B. van der Geer, M.J. de Loos, F.B. Kiewiet, M.J. van der Wiel, Physical Review Letters 93 (2004) 094802.

[22] B.W. Reed, JAP (in press 2006)

[23] G.H. Jansen, Journal of Applied Physics 84 (1998) 4549-4567.

[24] I. Langmuir, K.T. Compton, Rev. Mod. Phys. 3 (1931) 191-257.

[25] M. Merano, S. Collin, P. Renucci, M. Gatri, S. Sonderegger, A. Crottini, J.D. Ganiere, B. Deveaud, Rev. Sci. Inst. 76 (2005)

[26] J.F. Wishart, A.R. Cook, J.R. Miller, Rev. Sci. Inst. 75 (2004) 4359-4366.

[27] J.W. Lewellen, Proceedings of LINAC 2004 (2004) 842-846.

[28] P. Kruit, G.H. Jansen, Space Charge and Statistical Coulomb Effects, in Handbook of Charged Particle Optics, J. Orloff, Editor. 1997, CRC Press: Boca Raton. p. 275-318.

[29] M. Mkrtchyan, J.A. Liddle, L.R. Harriott, E. Munro, Solid State Technology 43 (2000) $241-+$. 
[30] H. Rose. personal communication, 2005.

[31] G.B. Olson, in Martensite, G.B. Olson, W.S. Owen, Editors. 1992, ASM International. p. $1-11$.

[32] M. Cormier, F. Claisse, J. Less-Common Metals 34 (1974) 181.

[33] M.S. Oh, J.Y. Lee, J.K. Park, Metall. and Mat. Trans. A 35A (2004) 3071.

[34] T.B. Brill, R.J. Karpowicz, J. Phys. Chem. 86 (1982) 4260-4265.

[35] A.K. Burnham, R.K. Weese, B.L. Weeks, J. Phys. Chem. 108 (2004) 19432-19441.

[36] B.F. Henson, L. Smilowitz, B.W. Asay, P.M. Dickson, J. Chem. Phys. 117 (2002) 37803788.

[37] L. Smilowitz, B.F. Henson, B.W. Asay, P.M. Dickson, J. Chem. Phys. 117 (2002) 37893798.

[38] U. Anselmitamburni , Z.A. Munir, J. Appl Phys. 66 (1989) 5039-5045.

[39] C. Michaelsen, K. Barmak, T.P. Weihs, J. Phys. D Appl. Phys. 30 (1997) 3167-3186.

[40] E. Ma, C.V. Thompson, L.A. Clevenger, K.N. Tu, Appl. Phys. Lett. 57 (1990) 1262.

[41] K.J. Blobaum, K. Van Heerden, A.J. Gavens, T.P. Weihs, Acta Mater. 51 (2003) 38713884.

[42] X.X. Huang, N. Hansen, N. Tsuji, 312 (2006) 249-251.

[43] R. Eelkema, M.M. Pollard, J. Vicario, N. Katsonis, B.S. Ramon, C.W.M. Bastiaansen, D.J. Broer, B.L. Feringa, Nature 440 (2006) 163-163.

[44] D.A. Heller, E.S. Jeng, T.K. Yeung, B.M. Martinez, A.E. Moll, J.B. Gastala, M.S. Strano, Science 311 (2006) 508-511.

[45] H.J. Dai, E.W. Wong, C.M. Lieber, Science 272 (1996) 523-526.

[46] M. Fiebig, K. Miyano, Y. Tomioka, Y. Tokura, Science 280 (1998) 1925-1928.

[47] A. Cavalleri, T. Dekorsy, H. H. W. Chong, J.C. Kieffer, R.W. Schoenlein, Phys. Rev. B 70 (2004) 161102.

[48] K. Sokolowski-Tinten, C. Blome, C. Dietrich, A. Tarassevic, D. von der Linde, A. Cavalleri, J.A. Squier, M. Kammler, Phys. Rev. Lett. 87 (2001) 225701.

[49] K. Sokolowski-Tinten, C. Blome, J. Blums, A. Cavalleri, M. Horn von, Hoegen, D. von der Linde, Nature 422 (2003) 287-289.

[50] A. Cavalleri, Cs. Toth, C.W. Siders, J.A. Squier, F. Raksi, P. Forget, J. C. Kieffer, Phys. Rev. Lett. 87 (2001) 237401.

[51] A. Cavalleri, H. Chong, S. Formaux, T. E. Glover, P. A. Heimann, B.S. Mun, H. Padmore, J.C. Kieffer, R.W. Schoenlein, Phys. Rev. B 69 (2004) 153106.

[52] M. Chollet, L. Guerin, N. Uchida, S. Fukaya, H. Shimoda, T. Ishikawa, K. Matsuda, T. Hasegawa, A. Ota, H. Yamochi, G. Saito, R. Tazaki, S. Adachi, S. Koshihara, Science 307 (2005) 86-89.

[53] S. Iwai, M. Ono, A. Maeda, H. Matsuzaki, H. Kishida, H. Okamoto, Y. Tokura, Phys. Rev. Lett. 91 (2003) 057401.

[54] T. Ogasawara, H. Okamoto, Y. Tokura, Phys. Rev. B 68 (2003) 035106. 


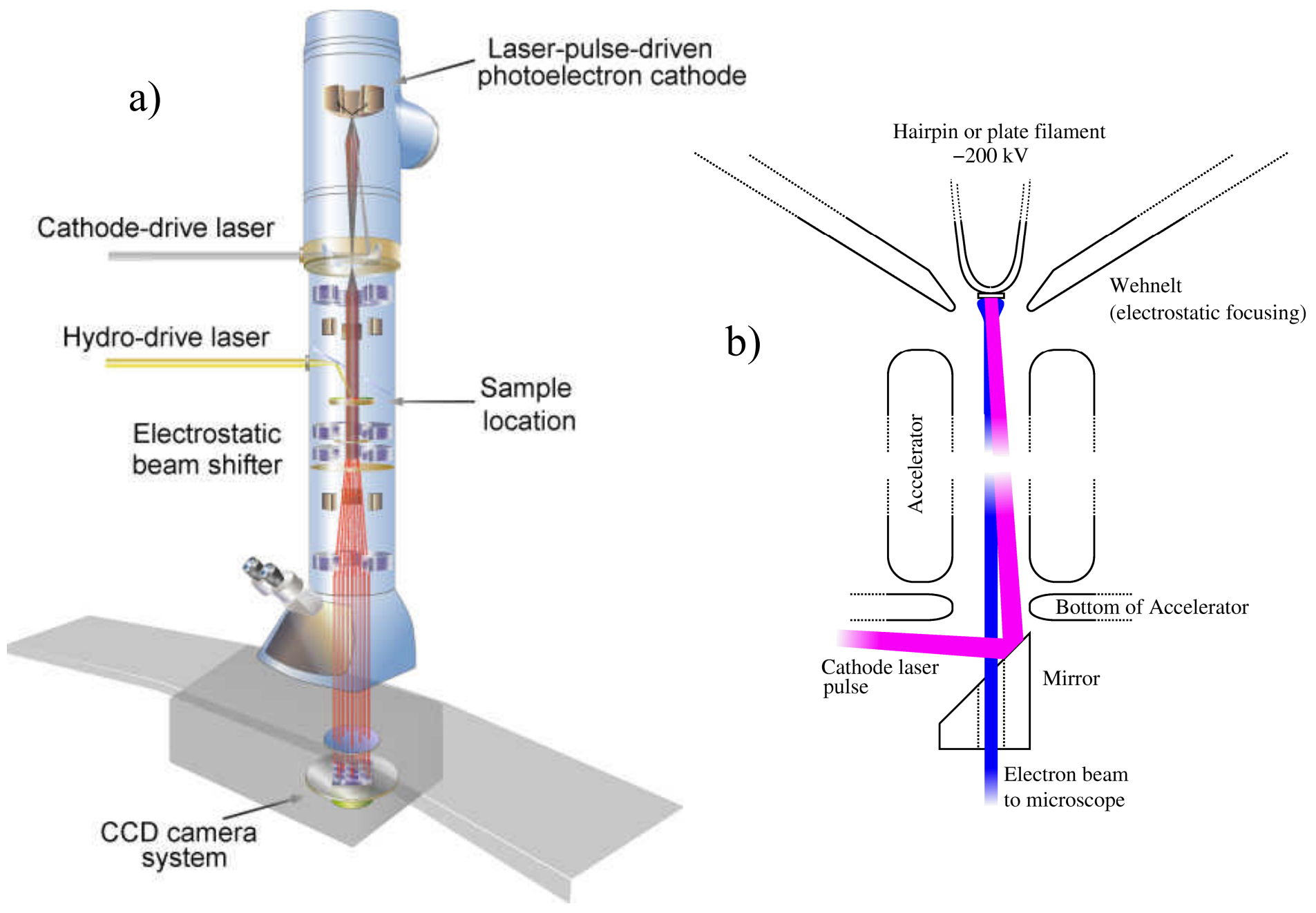

Figure 1: a) General schematic of DTEM and b) scheme for bringing photoexcitation pulse to the cathode. Fig. 1b is only schematic and not to scale. A section with a window and mirror has been inserted between the gun and condenser sections of the microscope to direct light from the cathode laser to the cathode. 


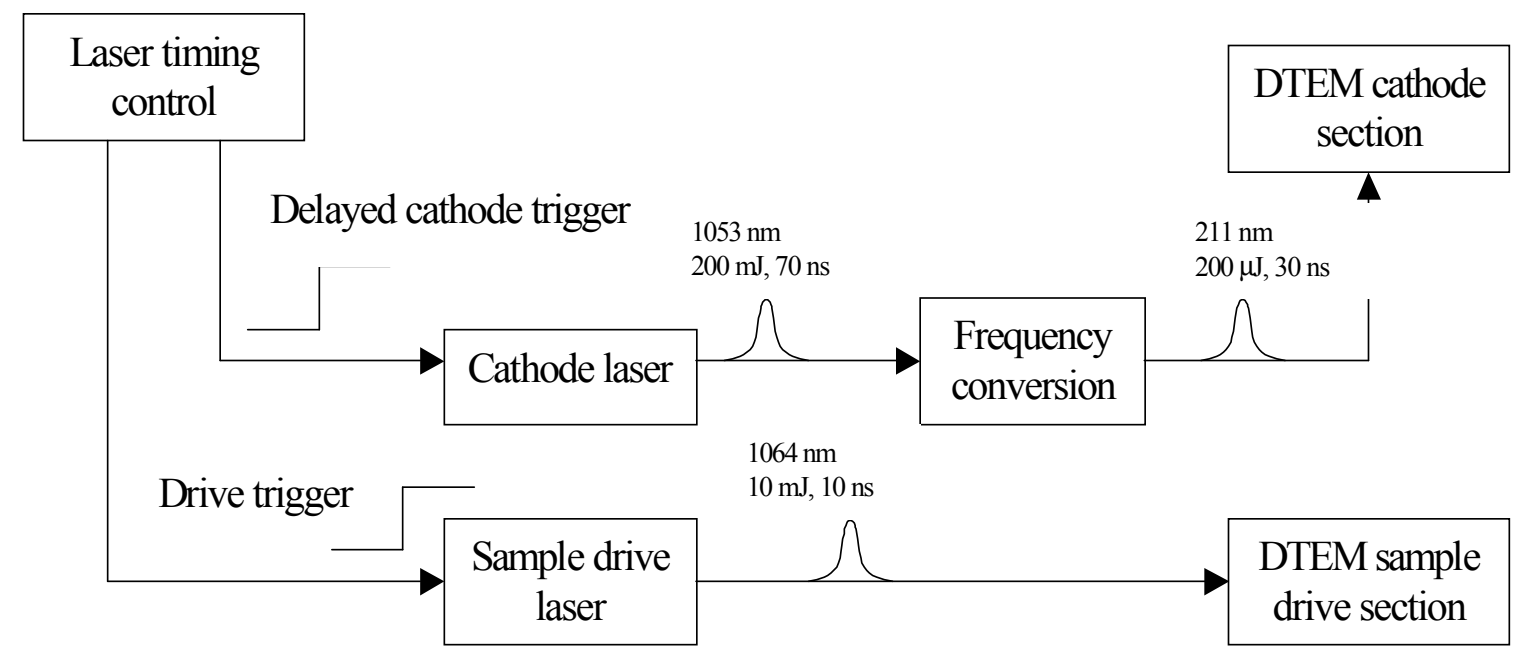

\section{Pulse sequence at sample}

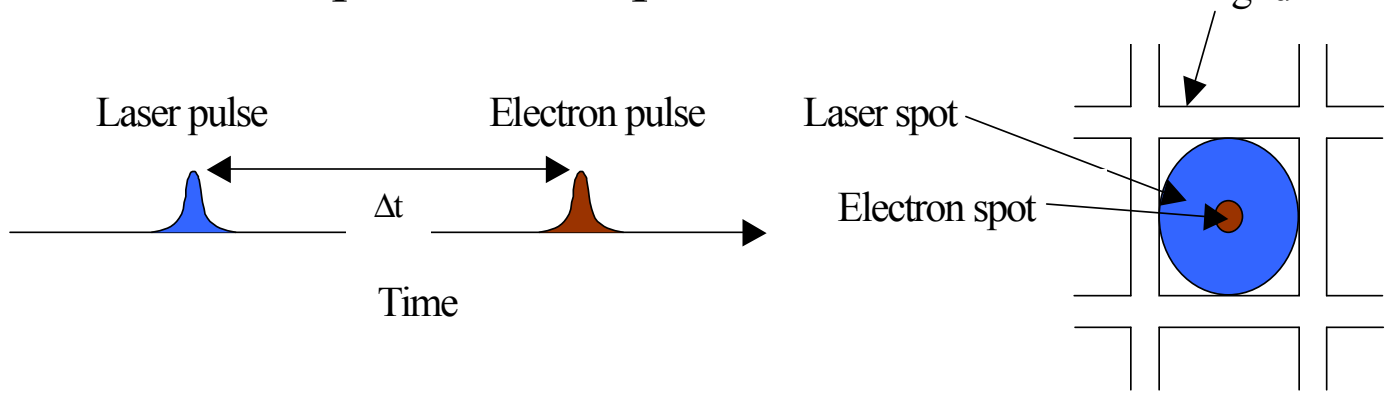

Sample region

Figure 2: Schematic of the DTEM laser system 


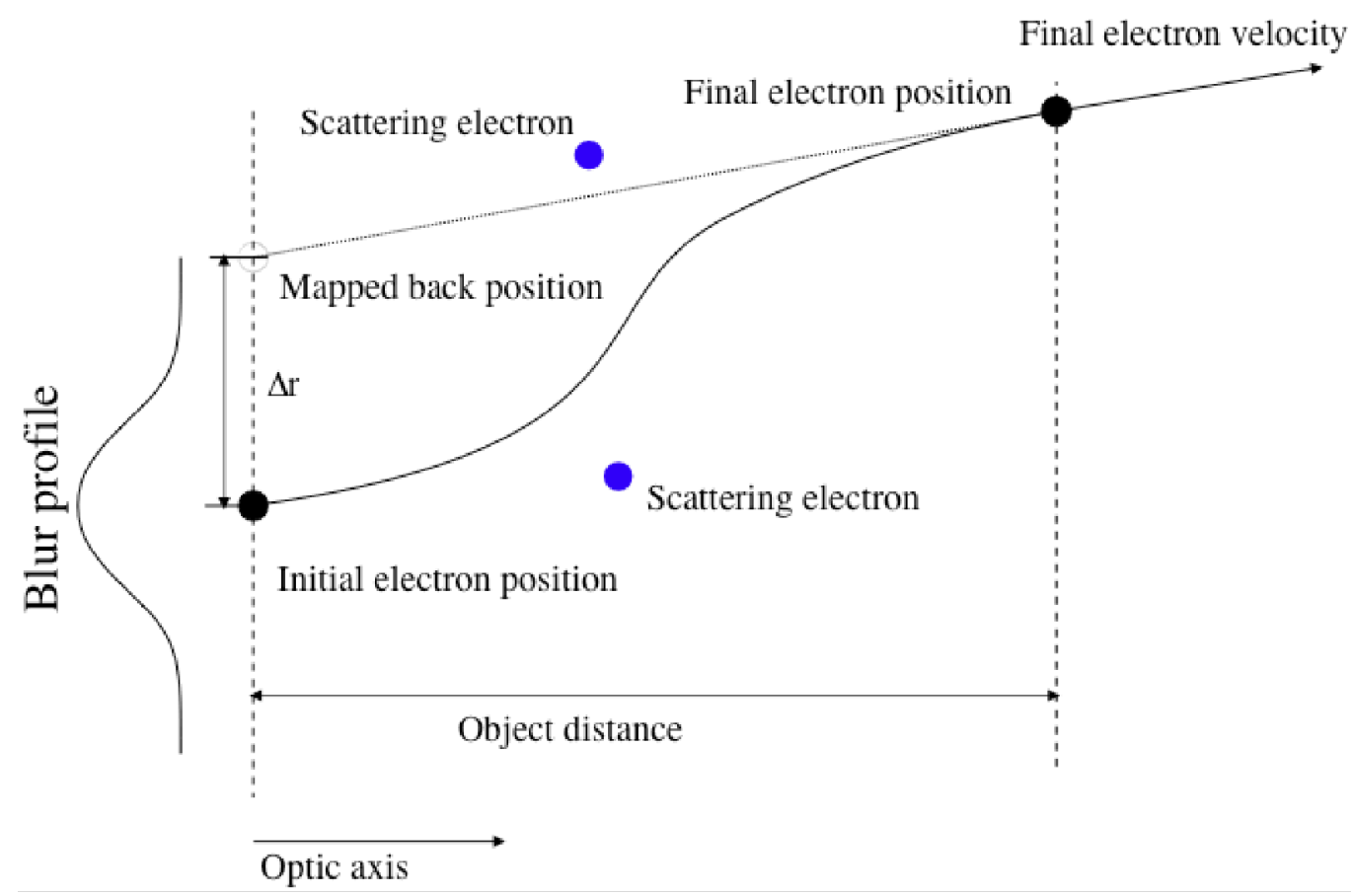

Figure 3: Blur is caused by random scattering of the trajectories of image forming electrons. 


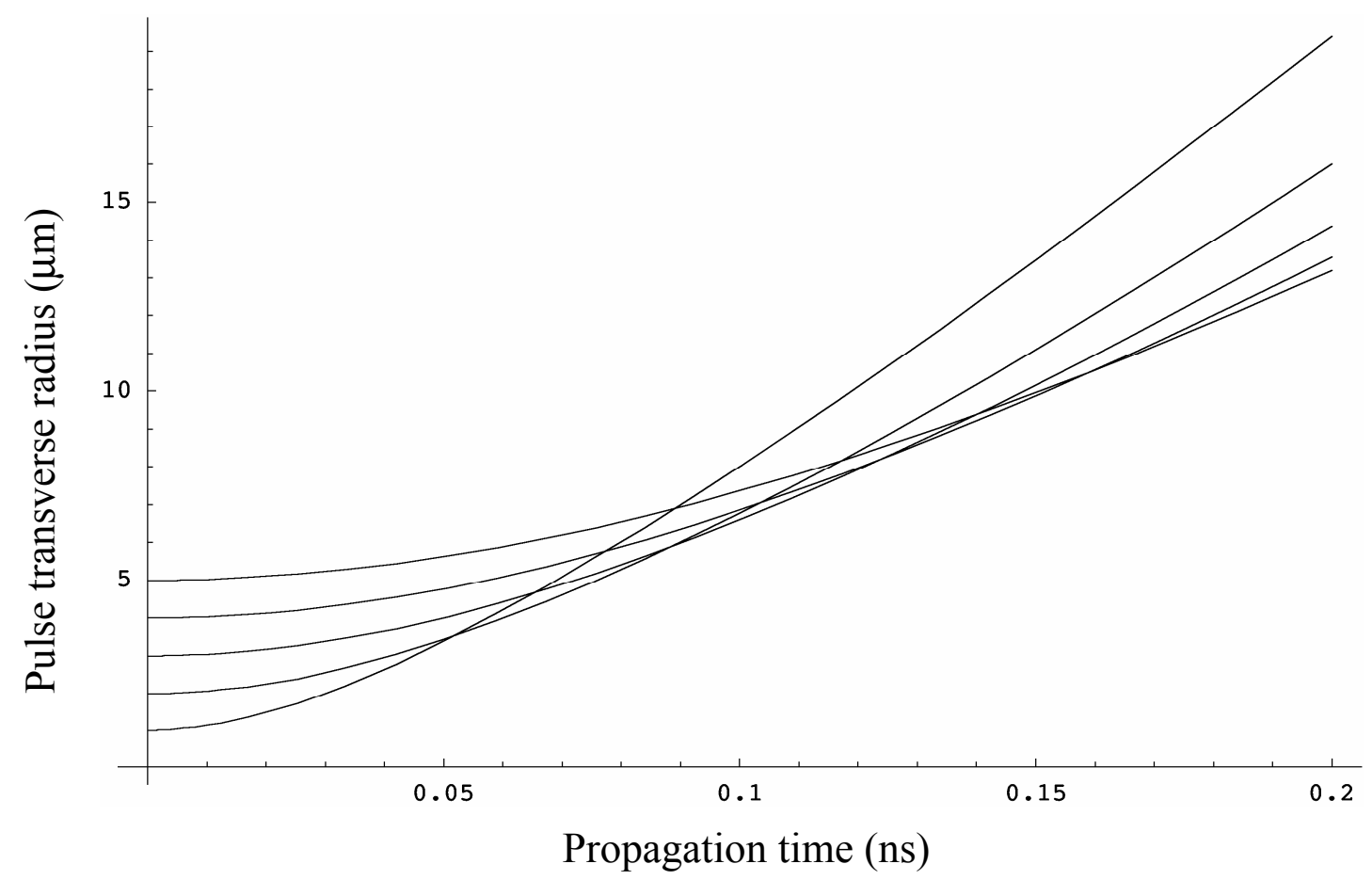

Figure 4: Calculated GSC expansion of a 1 ns duration uniform ellipsoidal pulse with $10^{6}$ electrons, initial radii raging from 1 to $5 \mu \mathrm{m}$. and no initial convergence. At $200 \mathrm{keV}, 1 \mathrm{~ns}$ of propagation time corresponds to about $20 \mathrm{~cm}$ pulse propagation distance. This model is the simplest that includes pulse expansion along the longitudinal and transverse directions, and provides a quick estimate of the magnitude of global space charge. 


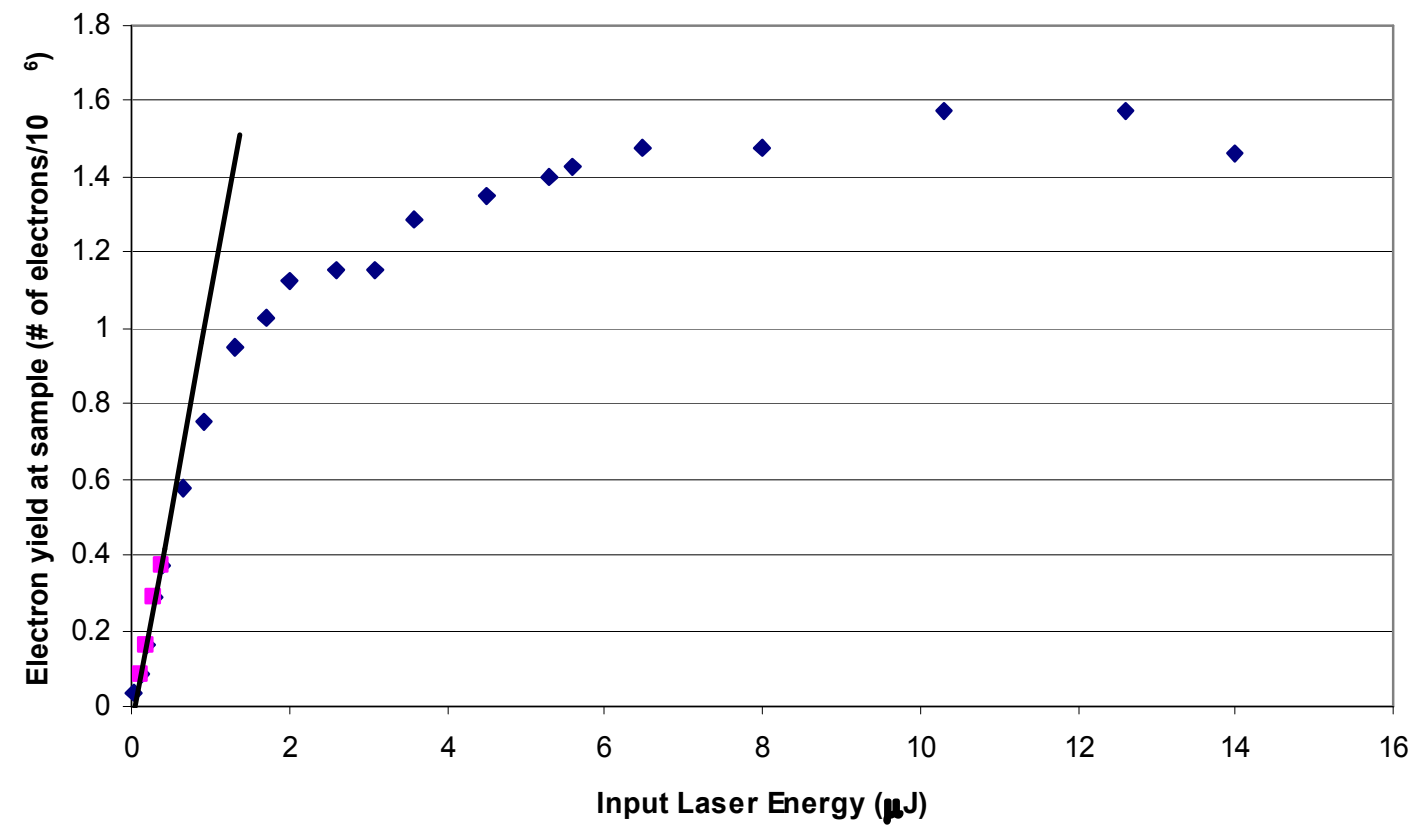

Figure 5: Electron yield per pulse at the sample as a function of laser pulse energy. The slope is extrapolated from the first few points in the graph, where the plot is linear. 


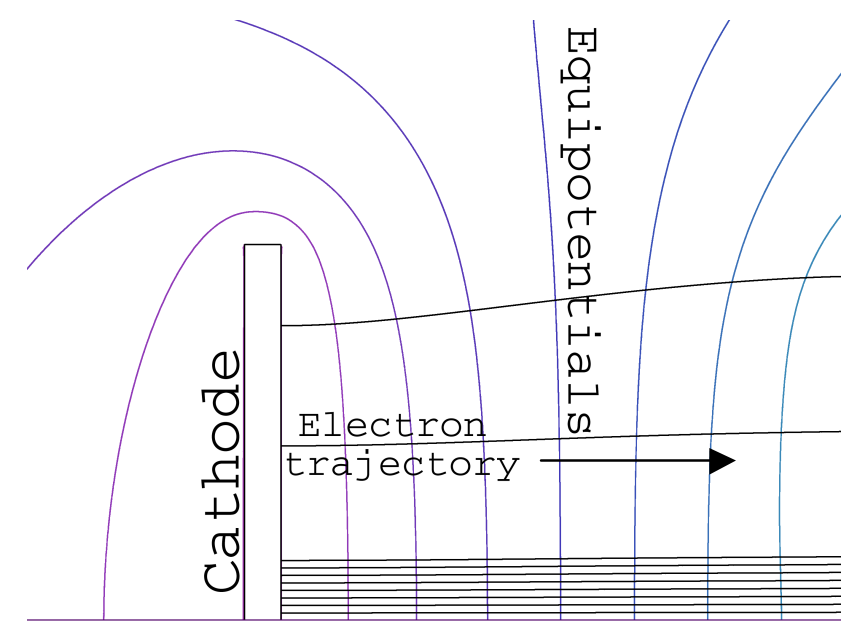

Figure 6: Model of electric field equipotentials and electron trajectories for a flat cathode embedded in a typical electron gun anode system. The horizontal axis is the propagation direction and the vertical axis is the radial direction for this cylindrically symmetric model.

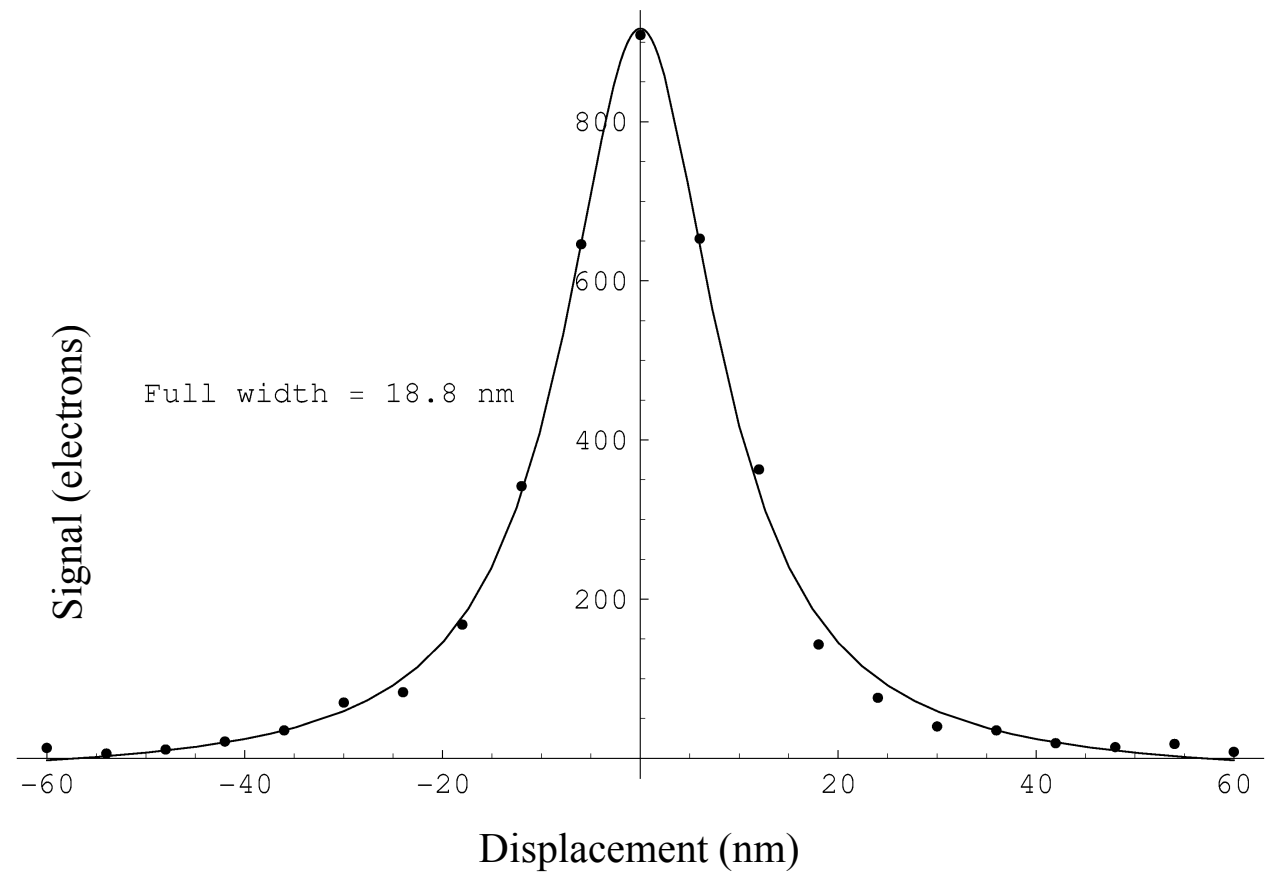

Figure 7: Blur profile for $20 \mathrm{k} 200 \mathrm{keV}$ electrons with a peak current corresponding to $10^{6}$ electrons in $1 \mathrm{~ns}$, assuming a crossover at the sample with a diameter of $1 \mu \mathrm{m}$ and $10 \mathrm{mrad}$ divergence half angle, propagated through one magnification stage with an objective focal length of $2.5 \mathrm{~mm}$ and $\sim 6.2 \mathrm{x}$ magnification. The fit is a Lorentzian. 


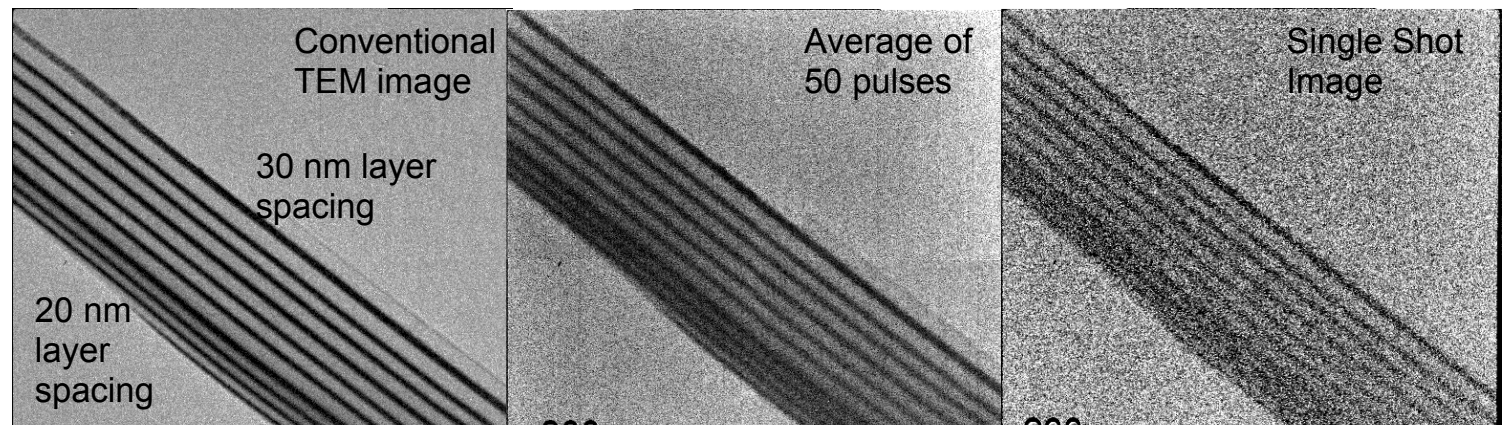

Figure 8: DTEM images obtained with a $35 \mathrm{~ns}$ pulse duration. The image on the left shows the sample under continuous illumination, the center image is the sum of $\mathbf{5 0}$ shots, and the image on the right is from a single shot.

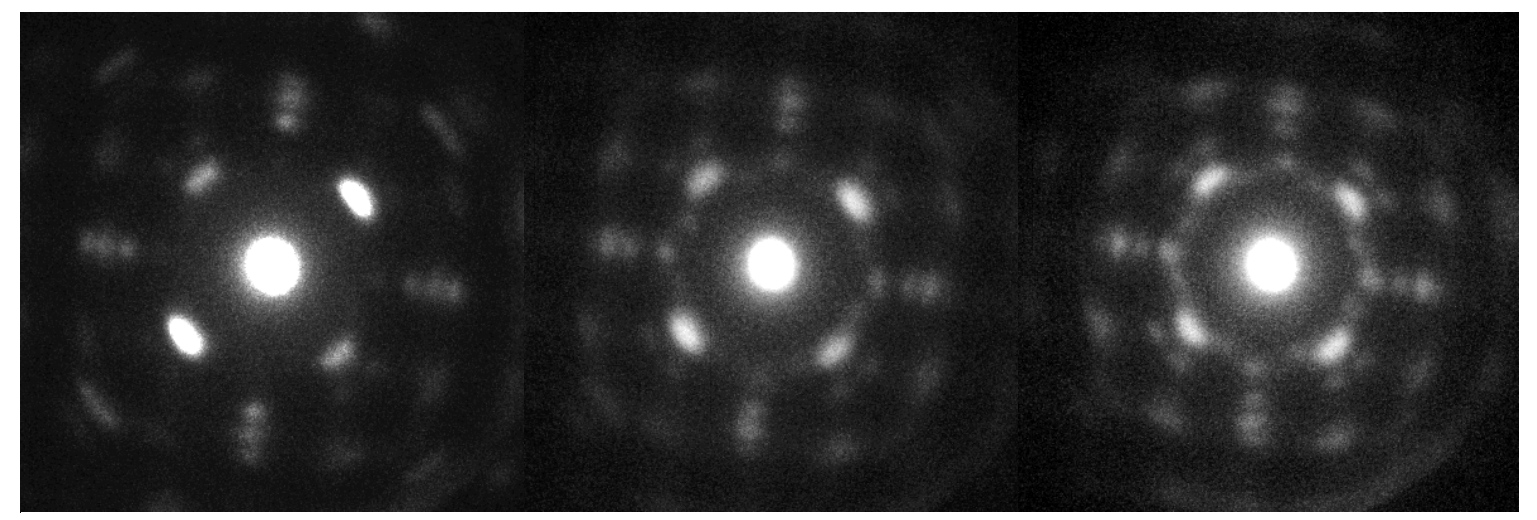

Figure 9:Diffraction pattern montage showing the evolution in the $\alpha \rightarrow \beta$ phase transformation with different pump/probe delays. 
$\beta$-HMX

Chair conformation Monoclinic
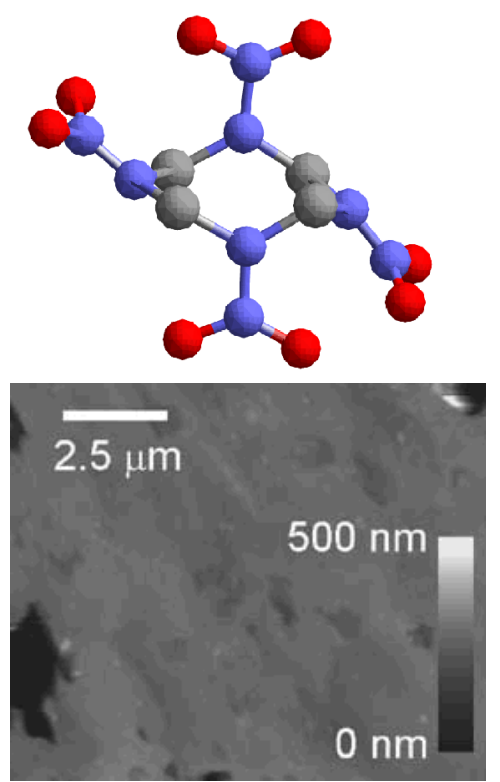

$\delta$-HMX

Boat conformation Hexagonal

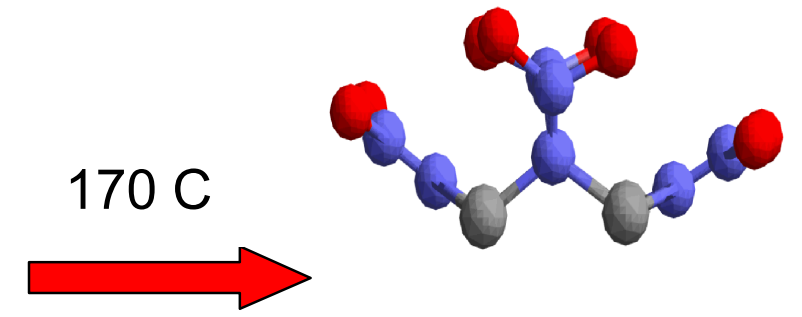

$7 \%$ Volume change

Figure 10:The chair to boat conformation change results in a monoclinic to hexagonal

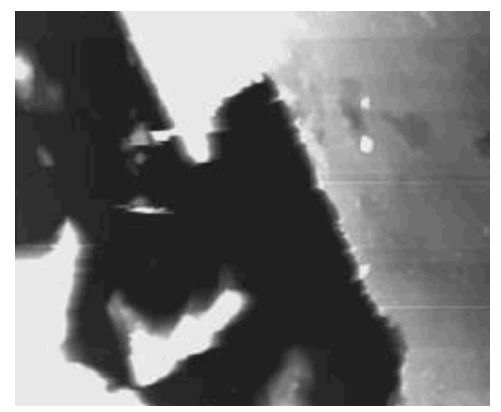

\section{phase change [11]}




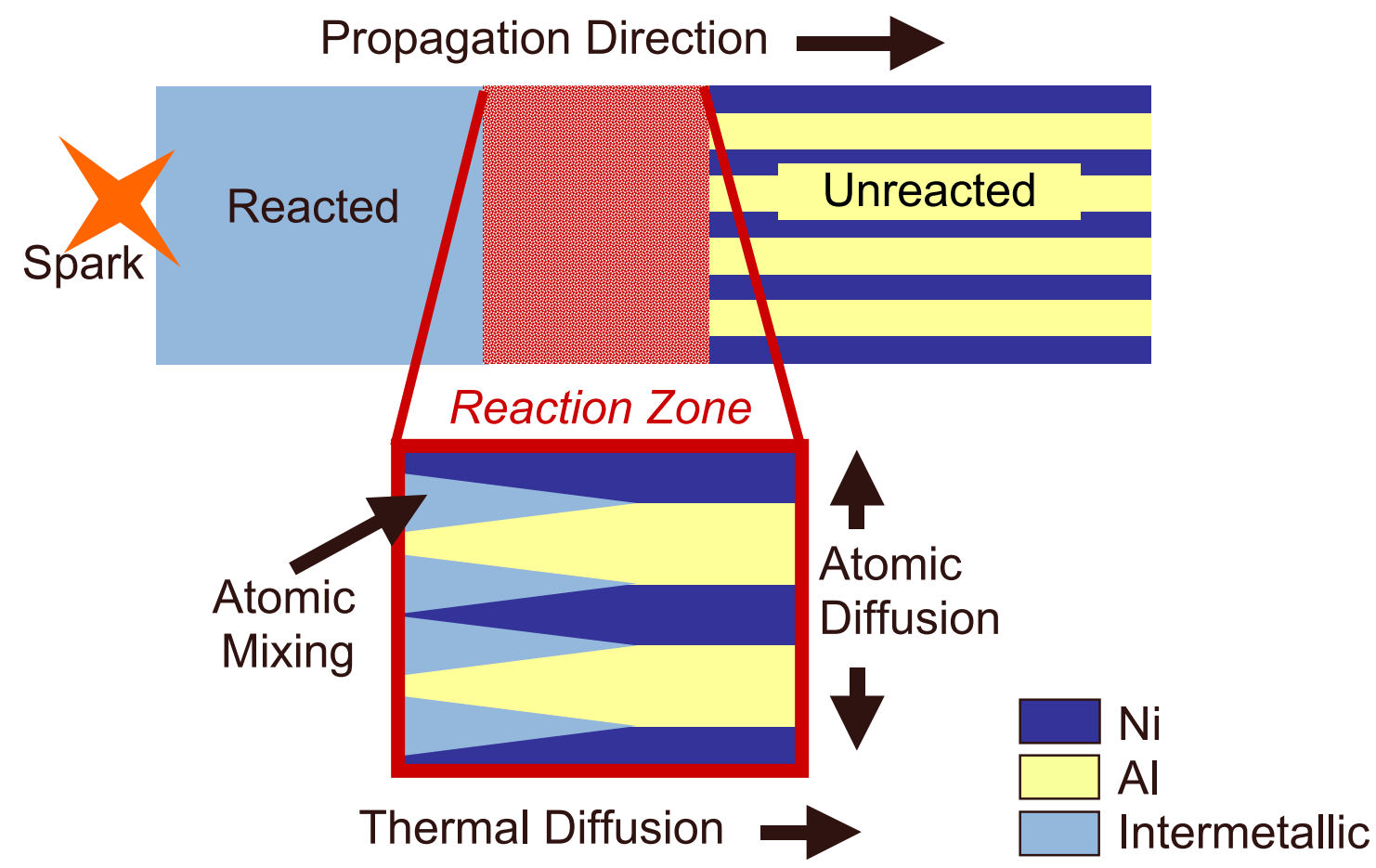

Figure 11: Schematic of interlayer diffusion and chemical reaction in a reactive multilayer foil. 